\title{
A necessary and sufficient condition for some steady Ricci solitons to have positive asymptotic volume ratio
}

\author{
Xiang Gao
}

\begin{abstract}
In this paper, we firstly establish a useful ODE relationship between $\mathrm{R}_{1}(\mathrm{c})$ and $\mathrm{V}_{1}(\mathrm{c})$ on the steady Ricci soliton. Based on this, we obtain a necessary and sufficient condition for some complete noncompact steady gradient Ricci solitons to have positive asymptotic volume ratio.
\end{abstract}

\section{Introduction and Main Results}

Recall that a complete Riemannian manifold $\left(M^{n}, g\right)$ is called a steady gradient Ricci soliton if there exists a smooth function $f: M^{n} \rightarrow \mathbb{R}$, called the potential function such that

$$
R_{i j}+\nabla_{i} \nabla_{j} f=0 .
$$

Moreover for the steady gradient Ricci soliton, we actually have

$$
R+|\nabla f|^{2}=C
$$

holds on $M^{n}$, where $C$ is a constant.

Key Words: steady Ricci soliton, asymptotic volume ratio, potential function.

2010 Mathematics Subject Classification: Primary 58C25; Secondary 35P05.

Received: March 2012.

Accepted: June 2012.

This work is supported by the Fundamental Research Funds for the Central Universities and NSFC11101267. 
In [2], it was proved by B.-L. Chen that the complete ancient solutions to the Ricci flow, and in particular the steady Ricci soliton, must have nonnegative scalar curvature. As a consequence, the potential function $f$ satisfies the following estimate:

$$
-\sqrt{C} r(x)+f(O) \leq f(x) \leq \sqrt{C} r(x)+f(O),
$$

where $r(x)$ denotes the distance function from $x$ to a fixed point $O$ in $M^{n}$.

Moreover, if we assume that the Ricci curvature is positive and the scalar curvature $R$ approaches 0 towards spatial infinity, then by the following Lemma 1.1 proved by H. X. Guo in [5], we can derive that there is one point where $R$ obtains its maximum, and the point of maximum is unique.

Lemma 1.1 (Guo). Let $\left(M^{n}, g\right)$ be a steady gradient Ricci soliton with positive (or negative) Ricci curvature, then there is at most one critical point of $R$.

Thus we can denote $O$ the unique point of maximum of $R$, called the origin, and assume $f(O)=0$ by adding a constant. Calculating the constant in (2) at $O$ we have

$$
R+|\nabla f|^{2}=R(O)=R_{0}
$$

Based on these, H. X. Guo [5] also proved a more precise estimate for the potential function of a complete steady gradient Ricci soliton as follows:

Theorem 1.2 (Guo). Assume $\left(M^{n}, g\right)$ is a complete steady gradient Ricci soliton with positive Ricci curvature, and the scalar curvature approaches 0 towards infinity. Then for any $\varepsilon>0$, there exists $r_{\varepsilon}>0$ such that when $r(x) \geq r_{\varepsilon}$ we have

$$
\left(\sqrt{R_{0}}-\varepsilon\right) r(x) \leq-f(x) \leq \sqrt{R_{0}} r(x),
$$

where $r(x)=d(x, O)$ and $R_{0}$ is the maximum of $R$.

Then define the functions

$$
\mathrm{V}: \mathbb{R} \rightarrow[0, \infty), \quad \mathrm{R}: \mathbb{R} \rightarrow[0, \infty)
$$

by

$$
\mathrm{V}(c)=\int_{\{f<c\}} d \mu, \quad \mathrm{R}(c)=\int_{\{f<c\}} R d \mu .
$$

In [1], the following ODE relating $\mathrm{V}(c)$ and $\mathrm{R}(c)$ was established for the shrinking Ricci soliton

$$
0 \leq \frac{n}{2} \mathrm{~V}(c)-\mathrm{R}(c)=c \mathrm{~V}^{\prime}(c)-\mathrm{R}^{\prime}(c) .
$$

In this paper, to prove our main result, we establish a similar result to (6) for the steady Ricci soliton as follows: 
Theorem 1.3. Define

$$
D_{1}(c)=\left\{x \in M^{n} \mid-f(x)<c\right\}, \quad \mathrm{R}_{1}(\mathrm{c})=\int_{D_{1}(c)} R d \mu, \quad \mathrm{V}_{1}(\mathrm{c})=\int_{D_{1}(c)} d \mu,
$$

then

$$
\mathrm{R}_{1}(\mathrm{c})+\mathrm{R}_{1}^{\prime}(\mathrm{c})-\mathrm{R}_{0} \mathrm{~V}_{1}^{\prime}(\mathrm{c})=0
$$

Recall that the asymptotic volume ratio (AVR) of a complete noncompact Riemannian manifold $\left(N^{n}, h\right)$ is defined by

$$
\operatorname{AVR}(h)=\lim _{r \rightarrow \infty} \frac{\operatorname{Vol}_{h} B(p, r)}{\omega_{n} r^{n}}
$$

if the limit exists, where $B(p, r)$ denotes the geodesic ball in $N^{n}$ with center $p$ and radius $r$ and $\omega_{n}$ is the volume of the unit Euclidean $n$-ball. It is easy to check that the $\operatorname{AVR}(h)$ is independent of the choice of $p$. Moreover, if $\left(N^{n}, h\right)$ has nonnegative Ricci curvature, then this limit (8) exists by the Bishop-Gromov volume comparison theorem.

For the case of shrinking Ricci solitons, H.-D. Cao and D.-T. Zhou [1] proved the following result aided by an observation of Munteanu [6].

Theorem 1.4 (Cao-Zhou). Any complete noncompact shrinking gradient linebreak Ricci soliton must have at most Euclidean volume growth, i.e.,

$$
\limsup _{r \rightarrow \infty} \frac{\operatorname{Vol} B(O, r)}{\omega_{n} r^{n}}<\infty
$$

For the case of steady Ricci solitons, by using Theorem 1.3, we can prove the following estimate.

Theorem 1.5. For the steady gradient Ricci soliton we have

$$
\mathrm{V}_{1}(\mathrm{c}) \geq \frac{\mathrm{R}_{1}(\mathrm{c})}{R_{0}}+\frac{R_{0} \mathrm{~V}_{1}\left(\mathrm{c}_{0}\right)-\mathrm{R}_{1}\left(\mathrm{c}_{0}\right)}{R_{0}} .
$$

In particular, more recently, observing the results in [1], [2], [4] and [8], B. Chow, P. Lu and B. Yang [3] derived a necessary and sufficient condition for noncompact shrinking Ricci soliton to have positive AVR as follows: 
Theorem 1.6 (Chow-Lu-Yang). Let $\left(M^{n}, g\right)$ be a complete noncompact shrinking gradient Ricci soliton, then $\operatorname{AVR}(g)$ exists (and is finite). Moreover, $\operatorname{AVR}(g)>0$ if and only if

$$
\int_{n+2}^{\infty} \frac{\mathrm{R}(c)}{c \mathrm{~V}(c)} d c<\infty
$$

In this paper, for the case of steady Ricci solitons, we prove a similar necessary and sufficient condition for some noncompact steady solitons to have positive AVR:

Theorem 1.7. Let $\left(M^{n}, g\right)$ be a complete noncompact steady gradient Ricci soliton such that the average scalar curvature

$$
0<\overline{\mathrm{R}}(g)=\lim _{r \rightarrow \infty} \frac{\int_{B(O, r)} R d \mu}{\operatorname{Vol}_{g}(B(O, r))}<\infty,
$$

then $\operatorname{AVR}(g)$ exists (and is finite). Moreover, $\operatorname{AVR}(g)>0$ if and only if

$$
\int_{c_{0}}^{\infty}\left(\frac{\mathrm{R}_{1}(c)}{\int_{0}^{c} \mathrm{R}_{1}(s) d s}-\frac{n+1}{c}\right) d c>-\infty .
$$

The paper is organized as follows. In section 2, we prove Theorem 1.3 by calculating, and then obtain Theorem 1.5 applying Theorem 1.3. Based on these, in section 3, we prove our main result Theorem 1.7.

\section{Proof of Theorem 1.3 and 1.5}

Proof of Theorem 1.3. Firstly, by Theorem 1.2, when $r(x)$ is greater than some constant $r_{\varepsilon}$, we have

$$
\left(\sqrt{R_{0}}-\varepsilon\right) r(x) \leq-f(x) \leq \sqrt{R_{0}} r(x) .
$$

Denote by

$$
D_{1}(c)=\left\{x \in M^{n} \mid-f(x)<c\right\} \quad \text { and } \quad \mathrm{V}_{1}(\mathrm{c})=\int_{D(c)} d V,
$$

then by the Co-Area formula (cf. [7]), we have

$$
\mathrm{V}_{1}(\mathrm{c})=\int_{0}^{c} d s \int_{\partial D_{1}(s)} \frac{1}{|\nabla(-f)|} d A
$$


Hence

$$
\mathrm{V}_{1}^{\prime}(\mathrm{c})=\int_{\partial D_{1}(c)} \frac{1}{|\nabla f|} d A
$$

Then taking the trace in

$$
R_{i j}+\nabla_{i} \nabla_{j} f=0
$$

we have

$$
R+\Delta f=0
$$

Thus by using the Divergence Theorem and (4)

$$
\begin{aligned}
-\int_{D_{1}(c)} R d \mu & =\int_{D_{1}(c)} \Delta f d \mu \\
& =\int_{\partial D_{1}(c)} \nabla f \cdot \frac{-\nabla f}{|\nabla f|} d A \\
& =-\int_{\partial D_{1}(c)}|\nabla f| d A \\
& =\int_{\partial D_{1}(c)} \frac{R-R_{0}}{|\nabla f|} d A \\
& =\int_{\partial D_{1}(c)} \frac{R}{|\nabla f|} d A-R_{0} \mathrm{~V}_{1}^{\prime}(\mathrm{c})
\end{aligned}
$$

Then by using the Co-Area formula again, we have

$$
\mathrm{R}_{1}(\mathrm{c})=\int_{D_{1}(c)} R d \mu=\int_{0}^{c} d s \int_{\partial D_{1}(s)} \frac{R}{|\nabla f|} d A .
$$

Hence

$$
\mathrm{R}_{1}^{\prime}(\mathrm{c})=\int_{\partial D_{1}(c)} \frac{R}{|\nabla f|} d A
$$

Therefore, we have

$$
-\mathrm{R}_{1}(c)=-\int_{D_{1}(c)} R d \mu=\int_{\partial D_{1}(c)} \frac{R}{|\nabla f|} d A-R_{0} \mathrm{~V}_{1}^{\prime}(\mathrm{c})=\mathrm{R}_{1}^{\prime}(\mathrm{c})-\mathrm{R}_{0} \mathrm{~V}_{1}^{\prime}(\mathrm{c}) .
$$

Now we turn to prove Theorem 1.5 by using Theorem 1.3. 
Proof of Theorem 1.5. Integrate the identity (7) from $c_{0}$ to $c$ we get

$$
\begin{aligned}
R_{0}\left(\mathrm{~V}_{1}(\mathrm{c})-\mathrm{V}_{1}\left(\mathrm{c}_{0}\right)\right) & =\int_{c_{0}}^{c} R_{0} \mathrm{~V}^{\prime}{ }_{1}(\mathrm{~s}) d \mathrm{~s} \\
& =\int_{c_{0}}^{c}\left(\mathrm{R}_{1}^{\prime}(\mathrm{s})+\mathrm{R}_{1}(\mathrm{~s})\right) d \mathrm{~s} \\
& =\mathrm{R}_{1}(\mathrm{c})-\mathrm{R}_{1}\left(\mathrm{c}_{0}\right)+\int_{c_{0}}^{c} \mathrm{R}_{1}(\mathrm{~s}) d \mathrm{~s}
\end{aligned}
$$

Therefore, (10) follows from the observation that $R_{1}(c)$ is nonnegative, because the scalar curvature $R \geq 0$.

\section{Proof of Theorem 1.7}

In this section, we prove Theorem 1.7 by using Theorem 1.2 and 1.3.

Proof of Theorem 1.7. Let

$$
\mathrm{P}(\mathrm{c})=\frac{\mathrm{R}_{1}(\mathrm{c})-R_{0} \mathrm{~V}_{1}(\mathrm{c})}{\mathrm{c}^{\mathrm{n}+1}} \text { and } \mathrm{N}(\mathrm{c})=\frac{\mathrm{R}_{1}(\mathrm{c})}{R_{0} \mathrm{~V}_{1}(\mathrm{c})},
$$

then

$$
\frac{\mathrm{N}(\mathrm{c})}{\mathrm{N}(\mathrm{c})-1}=\frac{\frac{\mathrm{R}_{1}(\mathrm{c})}{R_{0} \mathrm{~V}_{1}(\mathrm{c})}}{\frac{\mathrm{R}_{1}(\mathrm{c})}{R_{0} \mathrm{~V}_{1}(\mathrm{c})}-1}=\frac{\mathrm{R}_{1}(\mathrm{c})}{\mathrm{R}_{1}(\mathrm{c})-R_{0} \mathrm{~V}_{1}(\mathrm{c})}=\frac{\mathrm{R}_{1}(\mathrm{c})}{\mathrm{c}^{\mathrm{n}+1} \mathrm{P}(\mathrm{c})} .
$$

Note that $\frac{\mathrm{R}_{1}(\mathrm{c})}{\mathrm{V}_{1}(\mathrm{c})}$ is the average scalar curvature over the set $D(\mathrm{c})$, and the ODE (7) implies

$$
\begin{aligned}
\mathrm{P}^{\prime}(\mathrm{c}) & =\frac{\mathrm{R}_{1}^{\prime}(\mathrm{c}) \mathrm{c}^{\mathrm{n}+1}-(\mathrm{n}+1) \mathrm{R}_{1}(\mathrm{c}) \mathrm{c}^{\mathrm{n}}-R_{0} \mathrm{~V}_{1}^{\prime}(\mathrm{c}) \mathrm{c}^{\mathrm{n}+1}+(\mathrm{n}+1) R_{0} \mathrm{~V}_{1}(\mathrm{c}) \mathrm{c}^{\mathrm{n}}}{\mathrm{c}^{2 \mathrm{n}+2}} \\
& =\frac{\left(\mathrm{R}_{1}^{\prime}(\mathrm{c})-R_{0} \mathrm{~V}_{1}^{\prime}(\mathrm{c})\right) \mathrm{c}^{\mathrm{n}+1}-(\mathrm{n}+1) \mathrm{c}^{\mathrm{n}}\left(\mathrm{R}_{1}(\mathrm{c})-R_{0} \mathrm{~V}_{1}(\mathrm{c})\right)}{\mathrm{c}^{2 \mathrm{n}+2}} \\
& =\frac{-\mathrm{R}_{1}(\mathrm{c}) \mathrm{c}^{\mathrm{n}+1}-(\mathrm{n}+1) \mathrm{c}^{2 \mathrm{n}+1} \mathrm{P}(\mathrm{c})}{\mathrm{c}^{2 \mathrm{n}+2}} \\
& =\frac{-\frac{\mathrm{N}(\mathrm{c})}{\mathrm{N}(\mathrm{c})-1} \mathrm{c}^{2 \mathrm{n}+2} \mathrm{P}(\mathrm{c})-(\mathrm{n}+1) \mathrm{c}^{2 \mathrm{n}+1} \mathrm{P}(\mathrm{c})}{\mathrm{c}^{2 \mathrm{n}+2}} \\
& =-\left(\frac{\mathrm{N}(\mathrm{c})}{\mathrm{N}(\mathrm{c})-1}+\frac{n+1}{\mathrm{c}}\right) \mathrm{P}(\mathrm{c}) .
\end{aligned}
$$

Then we choose $c_{0}$ such that $\mathrm{P}\left(\mathrm{c}_{0}\right) \neq 0$, and integrate

$$
\mathrm{P}^{\prime}(\mathrm{c})=-\left(\frac{\mathrm{N}(\mathrm{c})}{\mathrm{N}(\mathrm{c})-1}+\frac{n+1}{\mathrm{c}}\right) \mathrm{P}(\mathrm{c}) \text {. }
$$


from $\mathrm{c}_{0}$ to $\mathrm{c}$ we have

$$
\mathrm{P}(\mathrm{c})=\mathrm{P}\left(\mathrm{c}_{0}\right) \mathrm{e}^{-\int_{\mathrm{c}_{0}}^{\mathrm{c}}\left(\frac{\mathrm{N}(\mathrm{c})}{\mathrm{N}(\mathrm{c})-1}+\frac{\mathrm{n}+1}{\mathrm{c}}\right) \mathrm{dc}} .
$$

From ODE (7) it is easy to see that

$$
\mathrm{R}_{1}(\mathrm{c})-R_{0} \mathrm{~V}_{1}(\mathrm{c})=-\int_{0}^{\mathrm{c}} \mathrm{R}_{1}(\mathrm{~s}) \mathrm{ds},
$$

which implies

$$
\frac{\mathrm{R}_{1}(\mathrm{c})}{\mathrm{c}^{\mathrm{n}+1} \mathrm{P}(\mathrm{c})}=\frac{\mathrm{N}(\mathrm{c})}{\mathrm{N}(\mathrm{c})-1}=\frac{\mathrm{R}_{1}(\mathrm{c})}{\mathrm{R}_{1}(\mathrm{c})-R_{0} \mathrm{~V}_{1}(\mathrm{c})}=-\frac{\mathrm{R}_{1}(\mathrm{c})}{\int_{0}^{c} \mathrm{R}_{1}(\mathrm{~s}) \mathrm{ds}}
$$

Note that (23) implies $\mathrm{P}(\mathrm{c}) \leq 0$. Furthermore, by the following bounds

$$
\left(\sqrt{R_{0}}-\varepsilon\right) r(x) \leq-f(x) \leq \sqrt{R_{0}} r(x)
$$

we have

$$
\begin{aligned}
\lim _{\mathrm{c} \rightarrow \infty} \mathrm{P}(\mathrm{c}) & =\lim _{\mathrm{c} \rightarrow \infty} \frac{\mathrm{R}_{1}(\mathrm{c})-R_{0} \mathrm{~V}_{1}(\mathrm{c})}{\mathrm{c}^{\mathrm{n}+1}} \\
& =\lim _{\mathrm{c} \rightarrow \infty} \frac{\mathrm{R}_{1}^{\prime}(\mathrm{c})-R_{0} \mathrm{~V}_{1}^{\prime}(\mathrm{c})}{(\mathrm{n}+1) \mathrm{c}^{\mathrm{n}}} \\
& =-\lim _{\mathrm{c} \rightarrow \infty} \frac{\mathrm{R}_{1}(\mathrm{c})}{(\mathrm{n}+1) \mathrm{c}^{\mathrm{n}}} \\
& =-\frac{1}{\mathrm{n}+1} \lim _{\mathrm{c} \rightarrow \infty} \frac{\mathrm{R}_{1}(\mathrm{c})}{\mathrm{V}_{1}(\mathrm{c})} \lim _{\mathrm{c} \rightarrow \infty} \frac{\mathrm{V}_{1}(\mathrm{c})}{\mathrm{c}^{\mathrm{n}}} \\
& =-\frac{1}{\mathrm{n}+1} \overline{\mathrm{R}}(g) \lim _{\mathrm{c} \rightarrow \infty} \frac{\operatorname{Vol}\left(B\left(O, \frac{c}{\sqrt{R_{0}}}\right)\right)}{\mathrm{c}_{n}} \\
& =-\frac{\omega_{n}}{(\mathrm{n}+1) R_{0}^{n / 2}} \overline{\mathrm{R}}(g) \operatorname{AVR}(g),
\end{aligned}
$$

that is to say

$$
-\frac{\omega_{n}}{(\mathrm{n}+1) R_{0}^{n / 2}} \overline{\mathrm{R}}(g) \operatorname{AVR}(g)=\lim _{\mathrm{c} \rightarrow \infty} \mathrm{P}(\mathrm{c}),
$$

which exists by (21). Since the average scalar curvature

$$
\overline{\mathrm{R}}(g)=\lim _{r \rightarrow \infty} \frac{\int_{B(O, r)} R d \mu}{\operatorname{Vol}_{g}(B(O, r))}>0,
$$

we have $\operatorname{AVR}(g)$ exists (and is finite). 
Moreover, by using (22) and (23) we have

$$
\begin{aligned}
\operatorname{AVR}(g) & =-\frac{(\mathrm{n}+1) R_{0}^{n / 2} \mathrm{P}\left(\mathrm{c}_{0}\right)}{\omega_{n} \overline{\mathrm{R}}(g)} \mathrm{e}^{-\int_{\mathrm{c}_{0}}^{\infty}\left(\frac{\mathrm{N}(\mathrm{c})}{\mathrm{N}(\mathrm{c})-1}+\frac{\mathrm{n}+1}{\mathrm{c}}\right) \mathrm{dc}} \\
& =-\frac{(\mathrm{n}+1) R_{0}^{n / 2} \mathrm{P}\left(\mathrm{c}_{0}\right)}{\omega_{n} \overline{\mathrm{R}}(g)} \mathrm{e}^{\int_{\mathrm{c}_{0}}^{\infty}\left(\frac{\mathrm{R}_{1}(\mathrm{c})}{\left.\int_{0}^{L_{1}(\mathrm{~s}) \mathrm{ds}}-\frac{\mathrm{n}+1}{\mathrm{c}}\right) \mathrm{dc}} .\right.}
\end{aligned}
$$

Note that $\mathrm{P}\left(\mathrm{c}_{0}\right)<0$, and by using $(12)$ we obtain that $\operatorname{AVR}(g)>0$ if and only if

$$
\int_{c_{0}}^{\infty}\left(\frac{\mathrm{R}_{1}(c)}{\int_{0}^{c} \mathrm{R}_{1}(s) d s}-\frac{n+1}{c}\right) d c>-\infty .
$$

\section{Acknowledgment}

I would especially like to thank the referee for meaningful suggestions that led to improvements of the article.

\section{References}

[1] H.-D. Cao, D. T. Zhou, On complete gradient shrinking Ricci solitons. J. Diff. Geom. 85, (2010), 175-185.

[2] B.-L. Chen, Strong uniqueness of the Ricci flow. J. Diff. Geom. 82, (2009), 363-382.

[3] B. Chow, P. Lu, B. Yang, A necessary and sufficient condition for Ricci shrinkers to have positive AVR. Proc. Amer. Math. Soc. 140 (2012), no. 6, 2179-2181.

[4] F.-Q. Fang, J.-W. Man, Z.-L. Zhang, Complete gradient shrinking Ricci solitons have finite topological type. C. R. Math. Acad. Sci. Paris 346, no. 11-12, (2008), 653-656.

[5] H. X. Guo, On the Ricci curvature of steady gradient Ricci solitons. J. Math. Anal. Appl. 363, (2010) 497-501.

[6] O. Munteanu, The volume growth of complete gradient shrinking Ricci solitons. arXiv: 0904. 0798. 
[7] R. Schoen, S.-T. Yau, Lectures on Differential Geometry. Conference Proceedings and Lecture Notes in Geometry and Topology, I. International Press, Cambridge, MA, (1994).

[8] S. J. Zhang, On a sharp volume estimate for gradient Ricci solitons with scalar curvature bounded below. Acta Math. Sinica 27, (2011), 871-882.

Xiang Gao,

School of Mathematical Sciences,

Ocean University of China,

Lane 238, Songling Road, Laoshan District, Qingdao City, Shandong

Province, 266100, People's Republic of China.

Email: gaoxiangshuli@126.com 